\title{
Utility of indocyanine green angiography in delaying breast reconstruction postmastectomy
}

\author{
Chu Luan Nguyen ${ }^{1,2,3}$ - $\cdot$ Yi Hui Angella Liu ${ }^{3} \cdot$ Tahmina Lata $^{3} \cdot$ Neshanth Easwaralingam ${ }^{1,2} \cdot$ Jue Li Seah $^{1}$. \\ Carina Chan ${ }^{3} \cdot$ Felicia Cao $^{3} \cdot$ Farhad Azimi $^{1} \cdot$ Cindy Mak $^{1} \cdot$ Carlo Pulitano $^{2,3} \cdot$ Sanjay Kumar Warrier ${ }^{1,3}$
}

Received: 16 November 2021 / Accepted: 2 February 2022 / Published online: 24 February 2022

(c) The Author(s) 2022

\begin{abstract}
Background Perfusion mapping with indocyanine green angiography (ICGA) in breast reconstruction can change intraoperative plans and reduce the risk of flap necrosis. We evaluated the utility of ICGA in the decision to delay reconstruction postmastectomy and its predictive power for necrosis.

Methods Single-institution retrospective study of implant-based breast reconstructions following mastectomy using ICGA from 2015 to 2021. A decision was made to proceed with immediate reconstruction or delay reconstruction based on clinical assessment and perfusion analysis. Incidence of complications in the immediate and delayed cohorts were assessed. Intraoperative ICGA perfusion values were correlated with postoperative outcomes to calculate diagnostic accuracy.

Results Three hundred twenty breast reconstructions were performed. Two hundred fifty-nine of these underwent immediate reconstruction, while 61 underwent delayed reconstruction due to poor perfusion. Median time between mastectomy and delayed reconstruction was 7.3 days (range, 4-21 days). All 8 cases (3.1\%) of necrosis were in the immediate cohort. Cases of necrosis had significantly lower intraoperative ICGA perfusion values compared to cases without necrosis (absolute values 13.1 versus 27.1 units, $p=0.017$ ). Increasing our ICGA cut-off score for necrosis from 14 to 22 units would have increased sensitivity from 63 to $100 \%$ but reduced specificity from 70 to $48 \%$.

Conclusions Delayed breast reconstruction due to poor perfusion on ICGA resulted in no cases of necrosis. A higher ICGA cut-off score for predicting necrosis is more sensitive but less specific. ICGA is useful as a supplement rather than a substitute for clinical assessment in flap perfusion evaluation.
\end{abstract}

Level of Evidence: Level III, Risk / Diagnostic Study.

Keywords Indocyanine green $\cdot$ Angiography $\cdot$ Breast $\cdot$ Reconstruction $\cdot$ Postmastectomy

\section{Introduction}

Breast reconstruction is a common procedure that can significantly improve quality of life for females who have undergone mastectomy. The success of the procedure is limited by mastectomy skin flap necrosis (MSFN) which has a reported

Chu Luan Nguyen

Chuluannguyen@gmail.com

1 Department of Breast Surgery, Chris O'Brien Lifehouse, Camperdown, 119-143 Missenden Rd, NSW 2050, Australia

2 Department of General Surgery, Royal Prince Alfred Hospital, Camperdown, 50 Missenden Rd, Camperdown NSW 2050, Australia

3 Department of Surgery, The University of Sydney, Sydney, Camperdown NSW 2050, Australia prevalence of up to $24 \%[1,2]$. This complication can be associated with infection, implant loss, reoperation, delayed adjuvant therapy, and poor cosmesis [3, 4].

The presence of MSFN after skin-sparing mastectomy (SSM) and nipple-sparing mastectomy (NSM) highlights the limitations of clinical assessment in evaluating tissue viability and the usefulness of an objective assessment tool [5-7]. Although indocyanine green angiography (ICGA) has been used in medical fields for years, its use in breast reconstruction surgery is relatively recent $[6,8]$. It involves real-time fluorescence imaging utilising a near-infrared camera after intravenous administration of the fluorophore, indocyanine green, to obtain an objective perfusion analysis [9].

Previous studies have shown that ICGA can assist with intraoperative decision-making involving excision of nonviable areas of mastectomy skin flaps to reduce risk of necrosis. 
They have also investigated the diagnostic accuracy of ICGA for predicting necrosis, but only in small cohorts [10-15]. We evaluated a more conservative approach in which reconstruction was delayed to a separate admission in cases with poorly perfused mastectomy skin flaps. The aim of this study was to assess the utility of ICGA in the decision to delay reconstruction and its diagnostic accuracy in a large cohort over 6 years of use.

\section{Material and methods}

This study was conducted in accordance with the Helsinki Declaration of 1964, and ethics was approved by the Human Research Ethics Committees (HREC) Sydney Local Health District, Australia (Protocol No: X21-0054 \& 2021/ETH00363). It was a single-institution retrospective cohort study of 320 implant-based breast reconstructions postmastectomy using ICGA routinely from February 2015, when it was first introduced into practice, to March 2021. Patients who underwent autologous breast reconstruction were excluded. Baseline patient and procedural characteristics were collected. Intraoperative information, including ICGA perfusion analyses, was collected. The incidence of complications, including MSFN, infection, dehiscence, implant loss, and unexpected reoperation, was documented. MSFN included epidermolysis, partial-thickness/ superficial necrosis, and full-thickness necrosis. Partial-thickness/ superficial necrosis was defined as loss of epidermis, partial dermal loss, and/ or eschar formation without subcutaneous fat exposure. Full-thickness necrosis was defined as loss of epidermis and dermis with exposure of subcutaneous fat, muscle, acellular dermal matrix, or implant [10].

\section{Surgical procedure}

All patients underwent SSM or NSM followed by breast reconstruction performed by one of four breast surgeons. Reconstruction was direct-to-implant or with tissue expander. All patients had electrocautery dissection, drains placed before skin closure and received preoperative antibiotics. No local anaesthetic or epinephrine-containing injections were used before or during surgery. After completion of mastectomy, laparotomy sponges were placed in the breast pocket to fill the dead space, the skin was closed temporarily using staples, and perfusion analysis was completed. A decision was made to either proceed with immediate reconstruction or delay reconstruction based on clinical assessment and the perfusion analysis. A delayed reconstruction involved closing the mastectomy skin flap wound and performing the implant-based reconstruction at a separate admission. Clinical assessment included evaluation of skin tissue colour, capillary refill, turgor, temperature, dermal edge bleeding, and skin flap thickness. ICGA analysis involved using qualitative images and an absolute perfusion value of 14 units as an indicator for poor perfusion.

\section{Indocyanine green angiography protocol}

Indocyanine green (ICG) was supplied as Infracyanine ${ }^{\circledR}$ $25 \mathrm{mg} / 10 \mathrm{~mL}$ (SERB, Paris, France). It was administered intravenously stat, at a weight-dependent dosage, just after the mastectomy was completed and prior to reconstruction. Fluorescence was excited by a near-infrared light source from the SPY Elite Fluorescence Imaging System (Novadaq Technologies Inc., Mississauga, Canada). Perfusion was video recorded up to $90 \mathrm{~s}$ after fluorescence was first detected in the mastectomy skin. The vascularization of the skin flap was analysed using perfusion maps and values obtained by SPY-Q Elite System software at the 90-s time point. Absolute perfusion values are based off a fixed grey scale that is consistent from image to image and were used during the intraoperative flap assessment. Relative perfusion values are based off a comparison with a selected area within the flap chosen as healthy tissue and were retrospectively analysed.

\section{Statistical analysis}

Differences between means were analysed using Student's $t$ test. Categorical variables were analysed using Fisher's exact tests. The predictive power of ICGA was calculated based on correlation of perfusion values to mastectomy skin flap outcomes. Sensitivity, specificity, positive predictive value, and negative predictive value were calculated for every perfusion reading associated with necrosis observed clinically. The results were ranked in numerical order to determine appropriate cut-off scores for necrosis [11]. A $p$ value $<0.05$ was considered significant. Receiver operating characteristic (ROC) curve analysis was performed to compare the ICGA absolute and relative perfusion feature's ability to predict postoperative necrosis observed clinically. Statistical analysis was performed with RStudio, version 1.4.1106.

\section{Results}

A total of 320 breast reconstructions (213 patients) were performed. Of these, 61 underwent a delayed reconstruction due to poor mastectomy skin flap perfusion based on clinical assessment and ICGA analysis. The remaining 259 breasts underwent an immediate reconstruction. 
Table 1 Baseline characteristics of delayed and immediate reconstruction cohorts

\begin{tabular}{llll}
\hline & $\begin{array}{l}\text { Delayed } \\
\text { reconstruction } \\
N=61,\end{array}$ & $\begin{array}{l}\text { Immediate } \\
\text { reconstruction, } \\
N=259\end{array}$ & $p^{*}$ \\
\hline Demographics & & & NS \\
Age, mean (SD) & $47.7(9.9)$ & $49.7(11.3)$ & \\
Any smoking, N (\%) & $3(4.9)$ & $27(10.4)$ & \\
Comorbidities, total, N (\%) & $7(11.5)$ & $24(9.3)$ & NS \\
Diabetes, N & 0 & $3(1.2)$ & \\
Tumour status, N (\%) & & $34(13.1)$ & \\
T1 & $2(3.3)$ & $85(32.8)$ & \\
$\geq$ T2 & $33(54.1)$ & $84(32.4)$ & \\
N0 & $22(36.1)$ & $39(15.1)$ & \\
$\geq$ N1 & $13(21.3)$ & $175(67.6)$ & \\
M0 & $42(68.9)$ & 0 & \\
M1 & $1(1.6)$ & $55(21.2)$ & \\
DCIS & $13(21.3)$ & $84(32.4)$ & \\
Prophylaxis & $18(29.5)$ & $69(26.6)$ & \\
Therapy & & $2(0.7)$ & \\
Any chemotherapy, N (\%) & $21(34.4)$ & $2(3.3)$ & \\
Any preop radiation, N & & & \\
(\%) & & & \\
Any hormonal therapy, & $11(18.0)$ & & \\
$\quad$ N (\%) & & & \\
\hline
\end{tabular}

*Fisher's exact test

$N S$ not significant at $p<.05, D C I S$ ductal carcinoma in situ, Preop preoperative

Comorbidities included hypertension, diabetes, asthma, thyroid disease, chronic kidney disease, ischaemic heart disease, atrial fibrillation

There was no statistically significant difference in age, smoking, comorbidities, tumour status, or therapy between the delayed and immediate reconstruction cohorts. The delayed cohort had a greater proportion of unilateral and tissue expander cases as well as greater mean mastectomy breast weight compared to the immediate cohort (Table 1). Mean intraoperative ICGA perfusion values for flaps in the delayed cohort were significantly lower than values for the immediate cohort (absolute values 6.9 versus 23.3 units; relative values $10.3 \%$ versus $30.7 \%, p<0.001$, respectively). For delayed reconstructions, mean ICGA perfusion values of their flaps at the delayed reconstruction were significantly improved compared to perfusion values at initial mastectomy (absolute values 42.0 versus 6.9 units; relative values $43.9 \%$ versus $10.3 \%, p<0.001$ respectively). The median time between the initial operation and delayed reconstruction was 7.3 days (range, 4-21 days).

There was no statistically significant difference in the complications of MSFN, infection, wound dehiscence, implant loss, or reoperation between the delayed and immediate cohorts (Table 2). Eight (3.1\%) breasts with partial-thickness and full-thickness necrosis were identified postoperatively in the immediate cohort compared to no cases in the delayed cohort. Cases of MSFN had significantly lower intraoperative ICGA perfusion values compared to cases without necrosis (absolute values 13.1 versus 27.1 units, $p=0.017$; relative values $21.0 \%$ versus $33.4 \%$, $p=0.049$ respectively) (Table 4 in the Appendix).

A cut-off score for necrosis of $\leq 14$ units predicted the development of MSFN in 5 out of 8 reconstructions. Associated sensitivity and specificity were $62.5 \%$ and $69.5 \%$, respectively. Necrosis was estimated to have been prevented in 3 of the 61 breasts (5\%) in the delayed cohort based on this cut-off score for necrosis and the corresponding positive predictive value of $5 \%$. When the cut-off perfusion score for predicting necrosis was increased to 22 units, sensitivity increased to $100 \%$ with a decrease in specificity to $48.2 \%$. Negative predictive value of ICGA was high while positive predictive value was relatively low (Table 3). Analysis of relative perfusion values showed that similar sensitivities and specificities could be obtained compared to absolute perfusion values (Table 5 in the Appendix). The ICGA absolute and relative perfusion units were both good predictors of postoperative necrosis with area under the ROC curves of 0.84 and 0.85 , respectively (Fig. 1).

\section{Discussion}

Identification of poorly perfused mastectomy skin flaps using ICGA helped guide intraoperative decisions to delay 61 cases which resulted in no cases of necrosis in this cohort. The overall rate of necrosis was $2.5 \%$ with 8 breasts complicated by necrosis, all of which were in the immediate reconstruction cohort. These numbers of necrosis are low compared to the literature especially with the inclusion of both partial-thickness and full-thickness necrosis cases [1, 2].

To our knowledge, our study is the first to evaluate the utility of ICGA in intraoperative decision-making to delay reconstruction in cases with poorly perfused flaps. Based on the ICGA perfusion analyses, the flaps in the delayed cohort were poorly perfused compared to flaps in the immediate cohort. Further ischemic insult from an implant or tissue expander in addition to ischemia sustained after the mastectomy may have placed the flaps in the delayed cohort at risk of necrosis. The flaps in the delayed cohort were already at greater risk of necrosis given the greater mean mastectomy breast weight compared to the immediate cohort $[6,16]$. The delayed cohort, however, did not amount any cases of necrosis. Delaying reconstruction in this cohort by a median of 7.3 days resulted in good outcomes. Three (5\%) of these breasts were prevented from developing necrosis based on an ICGA cut-off perfusion value of $\leq 14$ units. Although this 
Table 2 Surgery and outcomes of delayed and immediate reconstruction cohorts

\begin{tabular}{|c|c|c|c|}
\hline & $\begin{array}{l}\text { Delayed recon- } \\
\text { struction, } N=61\end{array}$ & $\begin{array}{l}\text { Immediate recon- } \\
\text { struction, } N=259\end{array}$ & $p^{*}$ \\
\hline \multicolumn{4}{|l|}{ Procedure, N (\%) } \\
\hline Unilateral & $37(60.7)$ & $95(36.7)$ & \multirow[t]{2}{*}{$<.001$} \\
\hline Bilateral & $24(39.3)$ & $164(63.3)$ & \\
\hline NSM & $48(78.7)$ & $177(68.3)$ & \multirow[t]{2}{*}{.366} \\
\hline SSM & $13(21.3)$ & $82(31.7)$ & \\
\hline Tissue expander reconstruction & $55(90.2)$ & $201(77.6)$ & \multirow[t]{2}{*}{$<.001$} \\
\hline Direct-to-implant & $6(9.8)$ & $58(22.4)$ & \\
\hline Sentinel lymph node biopsy & $31(50.8)$ & $131(50.6)$ & .395 \\
\hline Axillary dissection & $10(16.4)$ & $31(12)$ & .394 \\
\hline \multicolumn{4}{|l|}{ Intraoperative variables } \\
\hline Mastectomy breast weight, grams & 572.8 & 391.8 & .039 \\
\hline Implant size, mean cc (SD) & $358.0(74.7)$ & $360.0(124.5)$ & .605 \\
\hline Tissue expander size, mean cc (SD) & $474.1(110.3)$ & $408.4(116.3)$ & .390 \\
\hline Tissue expander fill ratio, mean \% (SD) & $58.1(20.8)$ & $63.4(20.5)$ & .150 \\
\hline ICGA absolute perfusion value, mean units (SD) & $6.9(5.5)^{* *}$ & $23.3(16.4)$ & $<.001$ \\
\hline ICGA relative perfusion value, mean $\%$ (SD) & $10.3(9.5)^{* *}$ & $30.7(18.8)$ & $<.001$ \\
\hline \multicolumn{4}{|l|}{ Complications, $\mathbf{N}(\%)$} \\
\hline Skin flap necrosis & 0 & $8(3.1)$ & .361 \\
\hline Infection & $3(4.9)$ & $16(6.2)$ & 1 \\
\hline Wound dehiscence & $2(3.3)$ & $10(3.9)$ & 1 \\
\hline Implant loss & $4(6.6)$ & $12(4.6)$ & .518 \\
\hline Return to theatre & $5(8.2)$ & $20(7.7)$ & 1 \\
\hline Haematoma & $1(1.6)$ & $3(1.2)$ & .573 \\
\hline Seroma & $2(3.3)$ & $14(5.4)$ & .746 \\
\hline
\end{tabular}

*Fisher's exact and Student's $t$ test

*** ICGA perfusion values during the initial operation, postmastectomy

$S S M$ skin-sparing mastectomy, NSM nipple-sparing mastectomy, ICGA indocyanine green angiography

\begin{tabular}{lllllllll}
\hline \multicolumn{7}{c}{ Cut-off ICGA absolute perfusion value, units } \\
\hline Statistical parameter, \% & $\mathbf{2 2}$ & $\mathbf{2 0}$ & $\mathbf{1 6}$ & $\mathbf{1 4}$ & $\mathbf{1 2}$ & $\mathbf{7}$ & $\mathbf{6}$ & $\mathbf{4}$ \\
Sensitivity & 100 & 87.5 & 62.5 & 62.5 & 50 & 37.5 & 25 & 12.5 \\
Specificity & 48.2 & 54.1 & 62.7 & 69.5 & 74.6 & 89.7 & 92.0 & 96.5 \\
Positive predictive value & 4.7 & 4.7 & 4.1 & 5.0 & 4.8 & 8.6 & 7.4 & 8.3 \\
Negative predictive value & 100 & 99.4 & 98.5 & 98.6 & 98.3 & 98.2 & 97.9 & 97.7 \\
\hline
\end{tabular}

ICGA indocyanine green angiography
Table 3 Cut-off indocyanine green angiography absolute values and associated sensitivity and specificity for predicting mastectomy skin flap necrosis is a small percentage, given that the rate of necrosis was low in our cohort, preventing even a small number of cases is arguably significant.

There are a small number of cohort studies in the literature that have investigated the diagnostic accuracy of ICGA for predicting necrosis [10-12, 14, 15, 17]. Munabi (2014) prospectively analysed 62 breasts that underwent autologous or implant-based breast reconstruction using ICGA. There was a $13 \%$ rate of necrosis. The excision of ischemic portions of mastectomy skin flaps was completed based on clinical assessment prior to ICGA. An absolute perfusion value of $\leq 7$ units predicted necrosis with $88 \%$ sensitivity and $83 \%$ specificity [11]. Similar results were obtained by Mattison (2016) who looked at the intraoperative difference between the surgeon's assessment of skin viability and ICGA assessment in 55 implant-based reconstructions. There was a $15 \%$ rate of necrosis. After mastectomy, the surgeon marked the area of skin flap to excise based on clinical assessment, and then ICGA was performed. The surface area of reduced perfusion was significantly greater with ICGA compared with the surgeon's assessment. An absolute perfusion value of 10 units had $100 \%$ sensitivity and $68 \%$ specificity [17]. In our 
Fig. 1 Receiver operating characteristic curve for indocyanine green angiography absolute (left) and relative (right) perfusion units. AUC, area under the curve
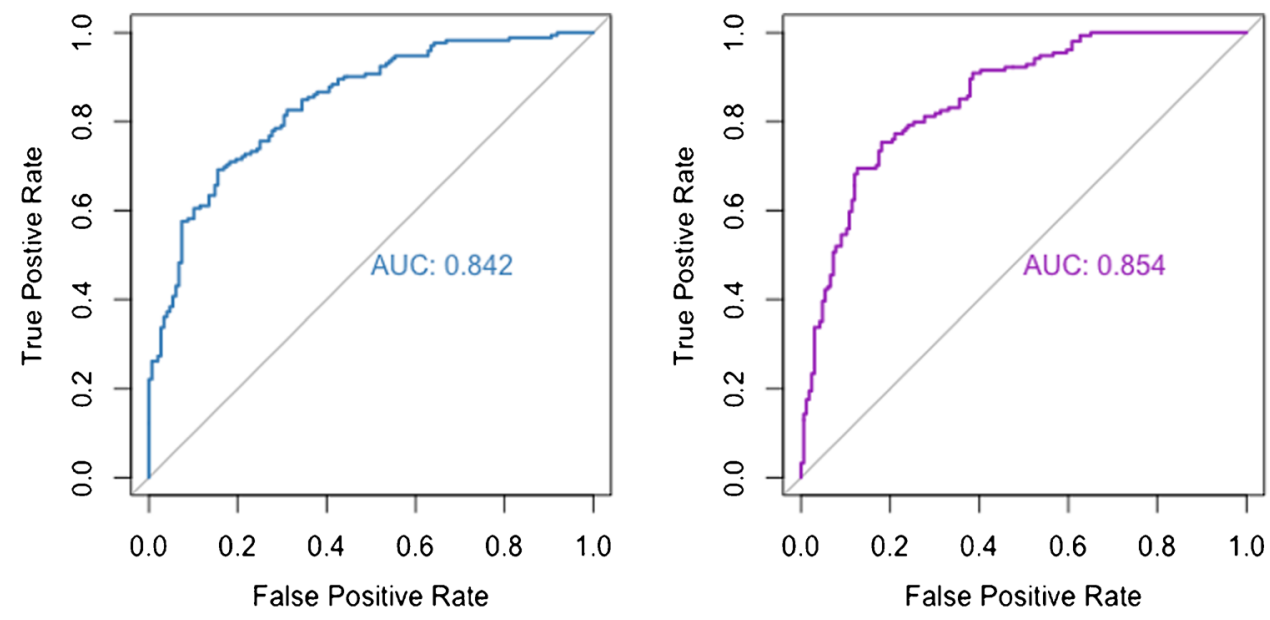

study, if a perfusion value of $\leq 10$ units was used as a cutoff, then sensitivity would be less than $50 \%$ and generate too many false-negatives to make it appropriate for clinical use.

We found that our sensitivities were comparable to these other studies if we used a higher perfusion cut-off score for necrosis. Their intraoperative decisions involved excision of ischemic portions of mastectomy skin flaps. We utilized a more conservative approach by delaying reconstruction altogether in cases with low perfusion values. Our intraoperative strategy may have contributed to the higher cut-off thresholds needed because our flaps were left intact. The excision of ischemic portions of a flap could result in higher measured perfusion of the flaps afterwards.

We found that ICGA could be highly sensitive but not specific at these levels. ICGA was much less specific for us compared to previous studies, which could be due to these studies having smaller sample sizes [11, 17]. The ICGA threshold we used for predicting necrosis was $\leq 14$ units which yielded a sensitivity of $63 \%$. Increasing our cut-off threshold would have increased sensitivity to $100 \%$ but at the expense of specificity. In other words, a perfusion value greater than the cut-off indicated a flap that was unlikely to develop necrosis. A perfusion value less than the cut-off, however, could incorrectly label cases as being at risk of necrosis resulting in false-positives.

Our negative predictive value for ICGA was high at over $98 \%$, while positive predictive value was only $5 \%$. Given that the rate of necrosis was low in our cohort, the positive predictive value was expected to be low, even when using a highly sensitivity test. In clinical practice, the usefulness of a test result for a patient depends on the prevalence of the disease in the population being tested. The diagnostic value of ICGA could possibly be improved if, based on history and clinical assessment, we limit its use to those patients at high risk for necrosis. A positive or a negative result is then more likely to be meaningful, than when the test is indiscriminately applied to patients.

There was a low incidence of MSFN overall in our experience using ICGA as an adjunct to clinical assessment. This retrospective analysis, however, highlights that a higher perfusion value threshold would have been more sensitive in predicting necrosis but at the expense of reduced specificity. ICGA may be most useful when used on patients at high risk of necrosis such as those with heavy mastectomy breast weight, high body mass index, and smokers. Preoperative radiotherapy was rare in our cohort and did not allow for accurate assessment of radiotherapy as a risk factor. Further studies to elucidate accurate thresholds and diagnostic accuracy in patients at high risk for necrosis could determine if ICGA should be reserved for these populations as opposed to routine use. Studies with larger sample sizes of delayed reconstructions based on ICGA could allow us to better evaluate this intraoperative strategy. This study was limited in that there was incomplete data available for certain patient comorbidities such as body mass index and intraoperative variables, such as the use of vasopressors, which may have affected the interpretation of ICGA perfusion values [11, $18]$.

\section{Conclusions}

ICGA can aid in assessment of perfusion for implant-based breast reconstructions. Delayed breast reconstruction due to poor perfusion on ICGA resulted in no cases of necrosis. A higher ICGA cut-off score for predicting necrosis is more sensitive but less specific. ICGA is useful as a supplement rather than a substitute for clinical assessment in flap perfusion evaluation. 


\section{Appendix}

Table 4 Variables associated with mastectomy skin flap necrosis
Table 5 Cut-off indocyanine green angiography relative values and associated sensitivity and specificity for predicting mastectomy skin flap necrosis

\begin{tabular}{|c|c|c|c|}
\hline & Necrosis, $N=8$ & No necrosis, $N=312$ & $\mathbf{p}^{*}$ \\
\hline Patient characteristics, $\mathbf{N}(\%)$ & & & NS \\
\hline Smoker & $1(12.5)$ & $29(9.3)$ & \\
\hline Diabetes & 0 & $3(1)$ & \\
\hline Procedure, $\mathrm{N}$ & & & NS \\
\hline Delayed reconstruction & 0 & $61(19.6)$ & \\
\hline Immediate reconstruction & $8(100)$ & $251(80.4)$ & \\
\hline SSM & $2(25)$ & $93(29.8)$ & \\
\hline NSM & $6(75)$ & $219(70.2)$ & \\
\hline Direct-to-implant & $3(37.5)$ & $61(19.6)$ & \\
\hline Tissue expander & $5(62.5)$ & $249(79.8)$ & \\
\hline \multicolumn{4}{|l|}{ ICGA perfusion values, mean (SD) } \\
\hline Absolute value at $90 \mathrm{~s}$, units & $13.1(7.0)$ & $27.1(21.8)$ & 0.017 \\
\hline Relative value at $90 \mathrm{~s}, \%$ & $21.0(10.2)$ & $33.4(20.8)$ & 0.049 \\
\hline Intraoperative variables, mean & & & NS \\
\hline Implant size, mean cc & 295.0 & 361.5 & \\
\hline Tissue expander fill ratio, $\%$ & 51.5 & 62.2 & \\
\hline Mastectomy breast weight, grams & 308.5 & 393.6 & \\
\hline
\end{tabular}

*Fisher's exact and Student's $t$ test.

$N S$ not significant at $p<0.05, S S M$ skin-sparing mastectomy, NSM nipple-sparing mastectomy.

\begin{tabular}{llllllll}
\hline Cut-off ICGA relative perfusion value, $\%$ & & & & & & \\
\hline Statistical parameter, $\%$ & $\mathbf{3 3}$ & $\mathbf{3 0}$ & $\mathbf{2 5}$ & $\mathbf{2 2}$ & $\mathbf{1 2}$ & $\mathbf{9}$ & $\mathbf{8}$ \\
\hline Sensitivity & 100 & 87.5 & 62.5 & 50 & 37.5 & 25.0 & 12.5 \\
Specificity & 42.8 & 47.6 & 59.2 & 65.0 & 86.5 & 93.2 & 95.5 \\
Positive predictive value & 4.3 & 4.1 & 3.8 & 3.5 & 6.7 & 8.7 & 6.7 \\
Negative predictive value & 100 & 99.3 & 98.4 & 98.1 & 98.2 & 98.0 & 97.7 \\
\hline
\end{tabular}

$I C G A$ indocyanine green angiography.
Funding Open Access funding enabled and organized by CAUL and its Member Institutions.

\section{Declarations}

Ethics approval This study was conducted in accordance with the Helsinki Declaration of 1964 and ethics was approved by the Human Research Ethics Committees (HREC) Sydney Local Health District, Australia (Protocol No: X21-0054 \& 2021/ETH00363).

Informed consent Consent was waived by HREC Sydney Local Health District.
Conflict of interest None of the authors have any conflict of interest in any of the products, devices, or drugs mentioned in this manuscript.

Open Access This article is licensed under a Creative Commons Attribution 4.0 International License, which permits use, sharing, adaptation, distribution and reproduction in any medium or format, as long as you give appropriate credit to the original author(s) and the source, provide a link to the Creative Commons licence, and indicate if changes were made. The images or other third party material in this article are included in the article's Creative Commons licence, unless indicated otherwise in a credit line to the material. If material is not included in the article's Creative Commons licence and your intended use is not permitted by statutory regulation or exceeds the permitted use, you will need to obtain permission directly from the copyright holder. To view a copy of this licence, visit http://creativecommons.org/licenses/by/4.0/. 


\section{References}

1. Woerdeman LAE, Hage JJ, Smeulders MJC, Rutgers EJT, van der Horst CMAM (2006) Skin-sparing mastectomy and immediate breast reconstruction by use of implants: an assessment of risk factors for complications and cancer control in 120 patients. Plast Reconstr Surg 118:321-330

2. Kobraei EM, Nimtz J, Wong L et al (2012) Risk factors for adverse outcome following skin-sparing mastectomy and immediate prosthetic reconstruction. Plast Reconstr Surg 129:234e-e241

3. Jeon FHK, Varghese J, Griffin M, Butler PE, Ghosh D, Mosahebi A (2018) Systematic review of methodologies used to assess mastectomy flap viability. BJS Open 2:175-184

4. Robertson SA, Jeevaratnam JA, Agrawal A, Cutress RI (2017) Mastectomy skin flap necrosis: challenges and solutions. Breast Cancer (Dove Med Press) 9:141-152

5. Komorowska-Timek E, Gurtner GC (2010) Intraoperative perfusion mapping with llaser-assisted indocyanine green imaging can predict and prevent complications in immediate breast reconstruction. Plast Reconstr Surg 125:1065-1073

6. Pruimboom T, Schols RM, Van Kuijk SM, Van der Hulst RR, Qiu SS. Indocyanine green angiography for preventing postoperative mastectomy skin flap necrosis in immediate breast reconstruction. Cochrane Database Syst Rev. 2020; 4:CD013280.

7. Driessen C, Arnardottir TH, Lorenzo AR, Mani MR. How should indocyanine green dye angiography be assessed to best predict mastectomy skin flap necrosis? A systematic review. J Plast Reconstr Aesthet Surg. 2020.

8. Singer R, Lewis CM, Franklin JD, Lynch JB (1978) Fluorescein test for prediction of flap viability during breast reconstructions. Plast Reconstr Surg 61:371-375

9. Reinhart MB, Huntington CR, Blair LJ, Heniford BT, Augenstein VA (2016) Indocyanine green: historical context, current applications, and future considerations. Surg Innov 23:166-175

10. Phillips BT, Lanier ST, Conkling $N$ et al (2012) Intraoperative perfusion techniques can accurately predict mastectomy skin flap necrosis in breast reconstruction: results of a prospective trial. Plast Reconstr Surg 129:778e-e788

11. Munabi NC, Olorunnipa OB, Goltsman D, Rohde CH, Ascherman JA (2014) The ability of intra-operative perfusion mapping with laser-assisted indocyanine green angiography to predict mastectomy flap necrosis in breast reconstruction: a prospective trial. J Plast Reconstr Aesthet Surg 67:449-455

12. Johnson AC, Colakoglu S, Chong TW, Mathes DW. Indocyanine green angiography in breast reconstruction: utility, limitations, and search for standardization. Plast Reconstr Surg Glob Open. 2020; 8:e2694.

13. Wang CY, Wang CH, Tzeng YS et al (2018) Intraoperative assessment of the relationship between nipple circulation and incision site in nipple-sparing mastectomy with implant breast reconstruction using the SPY imaging system. Ann Plast Surg 80:S59-S65

14. Phillips BT, Fourman MS, Rivara A. Comparing quantitative values of two generations of laser-assisted indocyanine green dye angiography systems: can we predict necrosis? Eplasty. 2014.

15. Newman MI, Jack MC, Samson MC (2013) SPY-Q analysis toolkit values potentially predict mastectomy flap necrosis. Ann Plast Surg 70:595-598

16. Ito H, Ueno T, Suga $\mathrm{H}$ et al (2019) Risk factors for skin flap necrosis in breast cancer patients treated with mastectomy followed by immediate breast reconstruction. World J Surg 43:846-852

17. Mattison GL, Lewis PG, Gupta SC, Kim HY (2016) SPY Imaging use in postmastectomy breast reconstruction patients: preventative or overly conservative? Plast Reconstr Surg 138:15e-21e

18. Moyer HR, Losken A (2012) Predicting mastectomy skin flap necrosis with indocyanine green angiography: the gray area defined. Plast Reconstr Surg 129:1043-1048

Publisher's Note Springer Nature remains neutral with regard to jurisdictional claims in published maps and institutional affiliations. 